\title{
The clinical prognostic significance of hs- cTnT elevation in patients with acute ischemic stroke
}

\author{
Lanying $\mathrm{He}^{1 * \dagger} \mathbb{D}$, Jian Wang ${ }^{1 *+}$ and Weiwei Dong ${ }^{2}$
}

\begin{abstract}
Background: Cardiac autonomic dysfunction caused by ischemic stroke might lead to an adverse outcome. Elevated high sensitivity cardiac troponin (hs-cTnT) is a marker of cardiac disease, it can elevate in acute stroke patients. The aim of the present study was to investigate association between serum hs-cTnT with prognosis among patients with acute ischemic stroke.

Methods: Five hundred and sixteen patients (mean age $66.19 \pm 10.11$ ) with acute ischemic stroke underwent a comprehensive clinical investigation and serum hs-cTnT activity test. All patients were followed up for 3 months. The prognosis was death or major disability (modified Rankin Scale score $\geq 3$ ) at 3 months after acute ischemic stroke.

Results: $22.87 \%$ (118/516) of patients had serum hs-cTnT elevation ( $\geq 14 \mathrm{ng} / \mathrm{l})$. Compared with normal hs-TnT group, the incidence of insular stroke (adjusted odds ratio, 2.84; 95\% confidence interval, $1.48-4.17 ; P=0.001$ ) were more likely in patients with hs-cTnT elevation. In fully adjusted models, there was an association between serum hscTnT elevation and death (adjusted odds ratio, 3.14; 95\% confidence interval, 1.16-8.49; $P=0.02$ ) and major disability(adjusted odds ratio, 2.07; 95\% confidence interval, 1.04-4.51; $P=0.04$ ), and composite outcome(adjusted odds ratio,2.22;95\% confidence interval, $1.10-4.48 ; P=0.03$ ).

Conclusions: Higher levels of serum hs-cTnT were independently associated with increased risk of death or major disability after stroke onset, suggesting that serum hs-cTnT may have prognostic value in poor outcomes of ischemic stroke.
\end{abstract}

Keywords: Acute ischemic stroke, Heart damage, Hs-cTnT,electrocardiographic abnormality, Insular stroke

\section{Background}

Stroke is an important contributor to death and major disability. Ischemic stroke is the most common subtype of stroke. Autonomic dysfunction is a frequent in stroke patients. Studies showed that cardiac autonomic dysfunction caused by ischemic stroke might lead to an adverse outcome. Cardiac comorbidities account for almost $20 \%$ of deaths after ischemic stroke, appropriate preventive or therapeutic measures can be taken if the patient with acute stroke at risk of myocardial injury could be identified at the time of admission. High

\footnotetext{
* Correspondence: 531324679@qq.com; 383376877@qq.com

'Lanying He and Jian Wang contributed equally to this work.

'Department of Neurology, The Second People's Hospital of Chengdu,

Chengdu 400010, People's Republic of China

Full list of author information is available at the end of the article
}

sensitivity cardiac troponin $\mathrm{T}$ (hs-cTnT) is the most sensitive marker of myocardial injury, however it can rise in several other conditions (e.g. renal failure, sepsis, heart failure, and pulmonary edema) $[1,2]$. In the last decade, the importance of hs-cTnT elevated in acute stroke has attracted many scholars' interest. Previous studies have shown that serum hs-cTnT is elevated in $10-30 \%$ of acute stroke patients [3-5]. In addition, some studies have found that elevated serum hs-cTnT levels may be associated with specific areas of brain damage [6]. However, it still remains unclear the pathomechanism of hs-cTnT elevation during the acute stage of ischemic stroke, which may be developed by neurally mediated autonomic dysregulation after acute stroke. In the other hand, in published studies, there were small numbers of

(C) The Author(s). 2018 Open Access This article is distributed under the terms of the Creative Commons Attribution 4.0 International License (http://creativecommons.org/licenses/by/4.0/), which permits unrestricted use, distribution, and 
patients. To date, whether hs-cTnT levels is associated with death or poor outcome remain uncertain $[7,8]$.

To test the hypothesis that if serum hs-cTnT levels can help predict cardiac complications and poor outcome in acute ischaemic stroke(AIS), we studied the prognostic correlates of elevated hs-cTnT levels on admission in a cohort of consecutive patients.

\section{Methods}

\section{Study population}

The consecutive patients who were admitted to the Second People's Hospital of Chengdu due to AIS within $72 \mathrm{~h}$ of symptom onset between May 2012 and December 2017. Stroke patients were diagnosed as AIS if the brain computed tomography (CT) scan was normal or showed acute ischemic changes according to World Health Organization definition (sudden neurological deficit with a putative vascular cause). Acute ischemic stroke was confirmed by diffusion-weighted imaging (DWI) magnetic resonance imaging (MRI) using Siemens Magnetom Avanto 1.5 Tesla (Siemens Medical Solutions, Erlangen, Germany). The severity of stroke was assessed by National Institutes of Health Stroke Scale score(NIHSS). Eligible patients were all patients admitted to our stroke unit during the study period. This study was approved by ethics committee. Written informed consent was obtained from all study participants or their legal proxies.

\section{Inclusion and exclusion criteria}

Patients were included in the study only if they fulfilled all the following criteria:1. Admission for first-ever acute ischemic stroke.2. Evidence of a single acute hemispheric lesion consistent with clinical manifestations. 3. Cardiac(include acute myocardial infarction, congestive heart failure, a history of tachyarrhythmia/bradyarrhythmia or atrial fibrillation),pulmonary disease and impaired renal function (estimated glomerularfiltration rate $<60 \mathrm{~mL} / \mathrm{min}$ per $1.73 \mathrm{~m}^{2}$ ) were excluded.4. Any pharmacological treatment, including $\beta$-blockers, possibly affecting the autonomic function were excluded. 5.Cerebral hemorrhage, fever, hypoxia also were excluded. No patients received mechanical thrombectomy and thrombolytic therapy. All patients received standard therapy, which consisted of aspirin, lipid-lowering medications and so on. All patients were followed up for 3 months. The outcome was defined as death and major disability (scores $3-5$ of modified Rankin Scale [mRS]) at 3 months after stroke onset.

\section{Data collection}

CT or MRI examination was conducted at the time of admission, and repeated examination was performed on 5 days after admission to confirm the location of the lesion. The presence of insular infarction was assessed by an experienced neuroradiologist blinded to clinical details.

Serum hs-cTnT was measured as part of routine laboratory testing on admission, which were measured by Elecsys and cobase analyzer (Roche diagnostics). Levels of serum hs-cTnT was considered abnormal if it was $\geq 14 \mathrm{ng} / \mathrm{l}$.

Standard 12-lead electrocardiogram (ECGs) examination was performed on admission and assessed by two inspectors, blinded for patients. The Data differences between observers were resolved by consensus.

After the heart investigation, if the patients were suspected to have acute coronary syndrome, the cardiologist would perform additional cardiac evaluations for the patients. The waveforms of 12-lead ECGs were uploaded in digitai form, and explained by a cardiologist. Two dimensional transthoracic echocardiography was performed on patients with suspected reversible cardiac ischemia, and then we excluded patients with reversible cardiac ischemia.

\section{Statistical analysis}

Firstly, patients were classified into normal and hs-cTnT elevation group according to the level of serum hs-cTnT on admission. Demographic characteristics, vascular risk factors, current smoke, and so on were compared between the 2 subgroups in univariate analysis, using Pearson $\times 2$ test, Fisher exact 2-sided test, or Student $t$ test, mean values( \pm standard deviation) were calculated for continuous variables. Mann-Whitney U test was used to test differences between two groups. We then performed logistic regressions analyses to determine the association between serum hs-cTnT and outcome(death, major disability and death/major disability), adjusting for age, sex, hypertension, current Smoking, current alcohol drinking, diabetes, hyperlipidemia, insular stroke, family history of stroke and NIHSS score. Results were expressed as adjusted odds ratios (OR) with the corresponding 95\% confidence interval (CI).The data were analyzed using SPSS software (SPASS 22.0). $P$ values $<0.05$ were considered as statistically significant.

\section{Results}

\section{Characteristics of the study subjects}

During the study period, 516 patients were identified, comprised $49.03 \%(253)$ men and 50.97\%(263) women, and the mean age was $66.19 \pm 10.11$ years( 38 -96 years). In the study population, 367 patients had a history of hypertension, 154 had a history of diabetes, 274 had a history of hyperlipidemia, 149 patients smoke,153patients current alcohol drinking. Of these patients, 152were diagnosed as insular stroke. During the 3-month follow-up period, 49 out of 516(9.49\%) patients had died. 
Univariable models for predictors of elevated hs-cTnT Serum hs-cTnT levels were normal in 398 (77.13\%) patients, the elevated hs-cTnT level in 118 (22.87\%). Baseline characteristics of patients in the normal hs-cTnT group $(<14 \mathrm{ng} / \mathrm{L})$ and the elevated hs-cTnT $\operatorname{group}(\geq 14 \mathrm{ng} / \mathrm{l})$ were compared (Table 1$)$. Patients with elevated hs-cTnT showed significantly higher prevalence of older age, insular stroke and high NIHSS score than patients with normal hs-cTnT (both $P<0.05$ ). In multivariate logistic regression, insular stroke associated with hs-cTnT elevation (adjusted odds ratio, 2.48; 95\% confidence interval, $1.48-4.17 ; P=0.00$ ).

\section{Multivariable models on the association between elevated hs-cTnT and prognosis}

Mortality rates were $24.58 \%(29 / 118)$ in the elevated hs-cTnT group at 3 months, which was significantly higher than that in the normal hs-cTnT group $(5.03 \%, 20 / 398) \quad(P=0.000)$. In addition to 49 dead, the remaining 467 patients, major disability rates were $40.45 \%(36 / 89)$ in the elevated hs-cTnT group at 3 months, which was significantly higher than that in the normal hs-cTnT group $(17.99 \%, 68 /$ 378) $(P=0.000)$. After adjusting for age, sex, hypertension, current smoking, current alcohol drinking, diabetes, hyperlipidemia, insular stroke, family history of stroke and NIHSS score on admission,3-month mortality in the elevated hs-cTnT group was higher than in the normal hs-cTnT group (adjusted odds ratio, 3.14; 95\% confidence interval, $1.16-8.49 ; P=0.02$ ), and 3-month major disability in the elevated hs-cTnT group was higher than in the normal hs-cTnT group (odds ratio, 2.17; 95\% confidence interval, 1.04-4.51; $P=0.04$ ). After adjusting for fully confounders, the combined prognosis of death/disability was significantly higher for the elevated hs-cTnT group at 3 months(adjusted odds ratio, 2.22; 95\% confidence interval, $1.10-4.48 ; P=0.03$ ).(Table 2).

In this study, we also found that the concentration of hs-cTnT were significantly correlated with poor prognosis, the higher the hs-cTnT, the worse the prognosis. The levels of hs-cTnT in the death group and the survival group were respectively $18.67 \pm 10.39,10.26 \pm 6.85(P=$ 0.00 ), the levels of hs-cTnT in the mRS $\leq 2$ group and the major disability group were respectively $9.14 \pm 5.98,14.14$ $\pm 8.15(\mathrm{P}=0.00)$, and the levels of hs-cTnT in the $\mathrm{mRS} \leq 2$ group and the composite outcome group were respectively $9.14 \pm 5.98,15.59 \pm 9.14(\mathrm{P}=0.00)$.

\section{Discussion}

Hs-cTnT is the most sensitive and specific biomarker of myocardial injury,which is widely used in the diagnosis of the patients with heart diseases, especially in patients with non-ST segment elevation acute coronary syndrome $[9,10]$. Many studies have shown that serum Hs-cTnT of many patients with acute stroke increases significantly. The current treatment guidelines for acute ischemic stroke patients recommend troponin evaluation in acute stage [11]. It is still controversial whether the increase of troponin after AIS is related to the mortality and disability rate of stroke patients. Most studies suggest that there is a link between them, but a few studies that hold the opposite view. Some studies have shown that elevated troponin is related to poor functional prognosis, and high troponin levels is associated with increased mortality [12-15]. The potential pathophysiological mechanism of troponin elevation in the AIS is still unclear, leading to considerable uncertainty in the diagnosis and treatment for the clinician. In our study, $22.87 \%(118 / 516)$ of acute stroke patients had elevated serum hs-cTnT level which was congruent with

Table 1 Comparison of baseline characteristics between patients with normal and elevated hs-cTnT group

\begin{tabular}{|c|c|c|c|c|}
\hline & hs-cTnT< 14 ng/L (398) & hs-cTnT $\geq 14$ ng/L (118) & $\mathrm{OR}(95 \% \mathrm{Cl})$ & $P^{*}$ \\
\hline Age, $y($ mean,SD $)$ & $65.36 \pm 10.07$ & $69.01 \pm 9.77$ & & 0.00 \\
\hline Females,n(\%) & $205(51.51)$ & $58(49.15)$ & $0.91(0.60-1.37)$ & 0.65 \\
\hline Men,n(\%) & 193(48.49) & $60(50.85)$ & $0.91(0.60-1.37)$ & 0.65 \\
\hline Hypertension,patients,n(\%) & 287(72.11) & $80(67.80)$ & $0.81(0.52-1.27)$ & 0.36 \\
\hline Current Smoking,n(\%) & $111(27.89)$ & $38(32.20)$ & $1.23(0.79-1.92)$ & 0.36 \\
\hline Current alcohol drinking,n(\%) & $115(28.89)$ & $38(32.20)$ & $1.17(0.75-1.82)$ & 0.49 \\
\hline Diabetes, n(\%) & $115(28.89)$ & $39(33.05)$ & $1.22(0.78-1.89)$ & 0.39 \\
\hline Hyperlipidemia,n(\%) & 214(53.77) & $60(50.85)$ & $0.89(0.59-1.34)$ & 0.58 \\
\hline $\mathrm{ST} / \mathrm{T}$ change, $\mathrm{n}(\%)$ & $110(27.64)$ & $39(33.05)$ & $1.293(0.83-2.01)$ & 0.23 \\
\hline Insular stroke,n(\%) & $89(22.36)$ & 63(53.39) & $3.98(2.58-6.12)$ & 0.00 \\
\hline Family history of stroke, n(\%) & $74(18,59)$ & $27(22.88)$ & $1.30(0.79-2.14)$ & 0.30 \\
\hline NIHSS score(min-max) & $2-25$ & $2-30$ & & \\
\hline NIHSS score(mean,SD) & $8.82 \pm 4.39$ & $11.80 \pm 5.21$ & & 0.00 \\
\hline
\end{tabular}

*Comparison between normal hs-cTnT group and elevated hs-cTnT group 
Table 2 Multivariable Models Showing Association Between elevated hs-cTnT and Prognosis

\begin{tabular}{lll}
\hline & OR $(95 \% \mathrm{Cl})$ & $\mathrm{P}^{*}$ \\
\hline death & $3.14(1.16-8.49)$ & 0.02 \\
Major disability(mRS 3-5) & $2.17(1.04-4.51)$ & 0.04 \\
Major disability(mRS3-5) + death & $2.22(1.10-4.48)$ & 0.03 \\
\hline
\end{tabular}

*Multivariable adjusted for age, sex, hypertension, current Smoking, current alcohol drinking, diabetes, hyperlipidemia, insular stroke, family history of stroke and NIHSS score

previous studies. Mortality rates and major disability rate in the elevated hs-cTnT group respectively were $24.58 \%, 30.31 \%$ at 3 months, which was significantly higher than that in the normal hs-cTnT group. After adjusting for fully confounders, we found a significant association of elevated hs-cTnT level with risks of death or major disability within 3 months after acute ischemic stroke. These results suggested this association was independent of established risk factors, including age, and baseline NIHSS score, and increased serum hs-cTnT elevation could be an independent risk factor of poor outcomes and have prognostic value for death or major disability among patients with acute ischemic stroke.

The evidence on whether increased troponin is associated with stroke was inconsistent: some studies had shown that damage to the right or left insular is associated with higher baseline troponin levels [16, 17], while others had not found any association between insular stroke and troponin levels [18, 19].In our study, 152 were diagnosed as insular stroke, patients with elevated hs-cTnT levels showed significantly higher prevalence of insular stroke, after adjusting for fully confounders, we found a significant association of insular stroke with elevated hs-cTnT level. These results suggested insular damage might contribute to cardiac autonomic dysfunction, the underlying pathophysiological mechanism might be the downregulation of parasympathetic activity, hence the relative up-regulation of sympathetic effects on cardiac function. As a result, this may lead to myocardial injury by contraction zone necrosis or ischemia. In our study showed that the range of hs-cTnT activity was between $14.46 \sim 58.51 \mathrm{ng} / \mathrm{L}$ in patients with increased hs-cTnT levels, which was much lower than that in the myocardial infarction patients. So, hs-cTnT elevation during the acute stage of ischemic stroke, which may be developed by neurally mediated autonomic dysregulation after acute stroke.

Some limitations of this study merit consideration. Firstly, in this study, we relied on a single baseline blood sample and thus we could not account for variations in serum hs-cTnT levels that occur over time, serum hs-cTnT levels should be measured repeatedly to allow longitudinal analysis, which might provide additional information on the development and on its prognostic implications. Secondly, We did not study that association of elevated serum hs-cTnT level with recurrent stroke, which might have an effect on the experimental results. Thirdly, although we adjusted for NIHSS score, which has been show to correlate with infarction volume, we lacked data on infarction volume. Fourth, we lack data on the possible influence of the left and right insular stroke on hs-cTnT and prognosis, respectively, because left and right insular lesion have different influence on the cardiac autonomic function.. In future experiments, we will avoid the aboved limitation, in order to obtain more reliable result.

\section{Conclusions}

Routine serum hs-cTnT measurement in patients with ischemic stroke may provide important novel clinical use. In addition, some studies should be encouraged in regarding to correct cardiac autonomic dysfunction and whether lowering hs-cTnT could prevent the poor outcome of ischemic stroke.

In conclusion, our findings indicated that higher levels of serum hs-cTnT in acute ischemic stroke were associated with increased risk of death or major disability at 3 months. Serum hs-cTnT may have potential predictive value in risk stratification of ischemic stroke.

\section{Abbreviations \\ Cl: Confidence Interval; hs-cTnT: High sensitivity cardiac troponin; M: Mean; OR: Odds Ratio; SD: Standard Deviation}

\section{Acknowledgments}

We thank all patients and their families for generously consenting to use of human tissues in this research.

\section{Funding}

This work was funded by the Health and Family Planning Commission of Chengdu (2015009), which is not involved in the database management (collection, analysis, interpretation of data) and has no access to patient information. The funding body did not participate in designing the study or writing the manuscript. The study protocol has undergone peer-review process by the funding body.

\section{Availability of data and materials}

The datasets used and/or analyzed during the current study are available from the corresponding author on reasonable request.

\section{Authors' contributions}

LYH was responsible for the concept and design of the study, data collection and analysis and the first draft of the paper and further manuscript. JW was responsible for concept and design of the study, the data analysis and interpretation. WWD was responsible for overseeing the concept and design of the study, the data analysis and interpretation, and writing the paper. All authors read and approved the final manuscript for publication.

\section{Ethics approval and consent to participate}

We obtained ethical approval for this study from the Medical and Health Research Ethics Committee in Second people's Hospital of Chengdu. The current study was carried out according to Declaration of Helsinki. Local legal and regulatory authorities as well as the medical secrecy will be followed. If the patient has consciousness disorder or aphasia, the decision cannot be made by themselves, the consent form can be signed by the patient's legal proxies. Prior to enrollment, each patients or their legal proxies will be given detailed information about the aims, scope and 
possible consequences of the trial by a physician. No diagnostic or interventional procedures required for the clinical trial. Written informed consent was obtained from all study participants or their legal proxies(516).

\section{Consent for publication}

Not applicable.

\section{Competing interests}

The authors declare that they have no competing interests.

\section{Publisher's Note}

Springer Nature remains neutral with regard to jurisdictional claims in published maps and institutional affiliations.

\section{Author details}

'Department of Neurology, The Second People's Hospital of Chengdu, Chengdu 400010, People's Republic of China. ${ }^{2}$ Department of Neurology, First Affiliated Hospital, Chongqing Medical University, ChongqingChina, 400030, People's Republic of China.

Received: 2 May 2018 Accepted: 6 August 2018

Published online: 20 August 2018

\section{References}

1. Jeremias A, Gibson CM. Narrative review: alternative causes for elevated cardiac troponin levels when acute coronary syndromes are excluded. Ann Intern Med. 2005:142(9): 786-791. [Free full text] [Pubmed].

2. Kelley WE, Januzzi JL, Christenson RH. Increases of cardiac troponin in conditions other than acute coronary syndrome and heart failure. Clin Chem. 2009:55(12): 2098-2112. doi: https://doi.org/10.1373/clinchem.2009. 130799. Epub 2009 Oct 8. [Free Article] [Pubmed].

3. Jensen JK, Kristensen SR, Bak S, Atar D, Hoilund-Carlsen PF, Mickley H. Frequency and significance of troponin T elevation in acute ischemic stroke. Am J Cardiol. 2007;99(1):108-12. https://doi.org/10.1016/j.amjcard.2006.10. 052.

4. Liu J, Wang D, Xiong Y, et al. High-sensitivity cardiac troponin T levels and risk of cerebral microbleeds in acute ischemic strokepatients with atrial fibrillation and/or rheumatic heart disease. J Neurol Sci. 2016;369:15-8. https://doi.org/10.1016/j.jns.2016.08.003. Epub 2016 Aug 2

5. Suleiman HM, Aliyu IS, Abubakar SA, et al. Cardiac troponin T and creatine kinase $M B$ fraction levels among patients with acute ischemic stroke in Nigeria. Niger J Clin Pract. 2017;20(12):1618-21. https://doi.org/10.4103/njcp. njcp_78_17.

6. Abdi S,Oveis-Gharan S, Sinaei F, Ghorbani A. Elevated troponin T after acute ischemic stroke: Association with severity and location of infarctin. Iran J Neurol., 2015,14(1):35-40. [ Free PMC Article][Pubmed].

7. Jensen JK, Atar D, Mickley H. Mechanism of troponin elevations in patients with acute ischemic stroke. Am J Cardiol. 2007;99(6):867-70. https://doi.org/ 10.1016/j.amjcard.2006.10.052

8. Freda BJ, Tang WH, Van LF, Peacock WF, Francis GS. Cardiac troponins in renal insufficiency: review and clinical implications. J Am Coll Cardiol. ,2002,40 (12): 2065-2071. [Free full text] [Pubmed].

9. Sayadnik M, Shafiee A, Jenab Y, Jalali A, Sadeghian S. Predictors of HighSensitivity Cardiac Troponin T Elevation in Patients with Acute Paroxysmal Supraventricular Tachycardia and Ischemic Heart Disease. Tex Heart Inst J,2017,44(5):306-311. DOl:https://doi.org/10.14503/THIJ-15-5338

10. Abiko M, Inai K, Shimada E, Asagai $S$, Nakanishi $T$. The prognostic value of high sensitivity cardiac troponin $\mathrm{T}$ in patients with congenital heart disease. J Cardiol,2018,71(4):389-393. DOI:10.1016/j.jjcc.2017.09.012.

11. Jauch EC, Saver JL, Adams HP, et al. Guidelines for the early management of patients with acute ischemic stroke: a guideline for healthcare professionals from the American Heart Association. American Stroke Association Stroke. 2013;44:870-947. https://doi.org/10.1161/STR.0000000000000158 Epub 2018 Jan 24.

12. Ahn $\mathrm{SH}$, Kim $\mathrm{YH}$, Shin $\mathrm{CH}$,et al. Cardiac Vulnerability to Cerebrogenic Stress as a Possible Cause of Troponin Elevation in Stroke. J Am Heart Assoc,2016,5(10):e004135. DOl:https://doi.org/10.1161/JAHA.116.004135[ Free PMC Article][Pubmed].

13. Scheitz JF, Mochmann HC, Erdur H, et al. Prognostic relevance of cardiac troponin $T$ levels and their dynamic changes measured with a highsensitivity assay in acute ischaemic stroke: analyses from the TRELAS cohort.
Int J Cardiol. 2014;177(3):886-93. https://doi.org/10.1016/j.jijcard.2012.01.055. Epub 2012 Feb 10

14. Faiz KW, Thommessen B, Einvik G, et al. Determinants of high sensitivity cardiac troponin T elevation in acute ischemic stroke.BMC Neurol 2014;14: 96. DOl:https://doi.org/10.1186/1471-2377-14-96.[ Free PMC Article][Pubmed].

15. Peddada K, Cruz-Flores S, Goldstein LB, et al. Ischemic stroke with troponin elevation: patient characteristics,resource utilization,and in-hospital outcomes. Cerebrovasc Dis,2016,42(2-3):213-223. DOl:https://doi.org/10. 1159/000445526.Epub 2016 May 3.

16. Song HS,Back JH,Jin DK,et al.Cardiac troponin T elevation after stroke: relationships between elevated serum troponin T,stroke location, and prognosis. J Clin Neurol,2008,4:75-83. [ Free PMC Article][Pubmed].

17. Scheitz JF, Endres M, Mochmann HC, et al. Frequency, determinants and outcome of elevated troponin in acute ischemic stroke patients. Int J Cardiol. 2012;157(2):239-42. https://doi.org/10.1016/j.ijcard.2012.01.055. Epub 2012 Feb 10

18. Christensen $\mathrm{H}$, Johannesen $\mathrm{HH}$, Christensen AF, et al. Serum cardiac troponin I in acute stroke is related to serum cortisol and TNF-alpha. Cerebrovasc Dis. 2004;18(3):194-9. https://doi.org/10.1159/000079941.

19. Barber M, Morton JJ, Macfarlane PW, et al. Elevated troponin levels are associated with sympathoadrenal activation in acute ischaemic stroke. Cerebrovasc Dis. 2007;23(4):260-6. https://doi.org/10.1159/000098325.

Ready to submit your research? Choose BMC and benefit from:

- fast, convenient online submission

- thorough peer review by experienced researchers in your field

- rapid publication on acceptance

- support for research data, including large and complex data types

- gold Open Access which fosters wider collaboration and increased citations

- maximum visibility for your research: over $100 \mathrm{M}$ website views per year

At $\mathrm{BMC}$, research is always in progress.

Learn more biomedcentral.com/submissions 